\title{
Nuclear Localization Signal
}

National Cancer Institute

\section{Source}

National Cancer Institute. Nuclear Localization Signal. NCI Thesaurus. Code C14089.

An amino acid sequence that directs the post-translational transport of a protein to the

nucleus. The sequence contains positively charged amino acids such as arginine and lysine. 